\title{
Control glicémico perioperatorio
}

\section{Perioperatory glycemic control}

\author{
Esteban Vallejo Agudelo1, Yerlin Andrés Colina Vargas, ${ }^{2, *}$, Jorge Alejandro Trujillo Zea ${ }^{3}$, \\ Heisa Catalina Durango Gómez ${ }^{4}$, Paulina Castro Echavarría ${ }^{5}$, Sara Rendón Villa ${ }^{6}$, Luis Fernando Botero Posada ${ }^{7}$ \\ Anestesiólogo. Clínica Universitaria Bolivariana/Universidad Pontificia Bolivariana. \\ Médico, Residente de Anestesiología. Universidad Pontificia Bolivariana. \\ Médico Residente de Anestesiología. Universidad CES \\ Neuroanestesiólogo Clínica Las Américas / Instituto Neurológico de Colombia INDEC \\ Médico Anestesiólogo, Universidad Pontificia Bolivariana. Medellín, Colombia. \\ 6 Anestesiólogo. Clínica Universitaria Bolivariana / Universidad Pontificia Bolivariana. Instituto Neurológico de Colombia INDEC. \\ Docente, Universidad Pontificia Bolivariana. \\ 7 Clínica universitaria Bolivariana.
}

Conflicto de intereses: No se tienen ningún tipo de conflicto de interés por parte de los autores.

Fecha de recepción: 28 de enero de 2021 / Fecha de aceptación: 25 de febrero de 2021

\begin{abstract}
Diabetes is the most common endocrinopathy, in $2014,8.6 \%$ of the population suffered from diabetes, and it was responsible for at least 3.7 million deaths per year. It is estimated that by that by 2050 more than $30 \%$ of the population will have this disease. In cardiovascular surgery, it is described that $5.2 \%$ of patients are undiagnosed diabetics and this rises to $10 \%-28 \%$ in non-cardiac surgeries. The adverse results are markedly high in those patients with poor glycemic control including an increase of more than $50 \%$ in mortality, as well as an increase in respiratory infections, surgical site infection, urinary infection, heart attack and acute kidney injury among others. During the preoperative period of patients with diabetes, it is important to review glycemic control and its current treatment, in addition to providing the patient instructions on how to adjust medications. Intraoperatively, any condition that leads to an uncontrolled increase in surgical stress must be controlled, since this in turn generates hyperglycemia. Knowledge of insulins, their pharmacology and schedules is essential to maintain blood glucose intraoperatively in normal ranges. Different practical algorithms are proposed for the correct and safe management of hyperglycemia in the perioperative period. All care should be continued in the postoperative period defining the continuity of the insulin therapies established and the postoperative care of the patient.
\end{abstract}

Key words: Perioperative period, diabetes mellitus, hypoglycemic agents, insulin, hyperglycemia, hypoglycemia.

\section{RESUMEN}

La diabetes es la endocrinopatía más común, en 2014, el 8,6\% de la población padecía diabetes siendo responsable de 3,7 millones de muertes por año. Se estima que para el 2050 más del 30\% de la población tendrá diabetes. En cirugía cardiovascular el 5,2\% de los pacientes son diabéticos no diagnosticados, cifra que aumenta hasta 10\%-28\% en cirugías no cardíacas. Los resultados adversos son marcadamente elevados en aquellos pacientes con mal control incluyendo un aumento del $50 \%$ en la mortalidad, así mismo, incremento de infecciones respiratorias, infección del sitio quirúrgico, infección urinaria, infarto agudo de miocardio y lesión renal aguda, entre otros. Durante el preoperatorio de pacientes con diabetes, es importante revisar el control glucémico y su tratamiento, además de proporcionar al paciente instrucciones por escrito sobre cómo ajustarlo. En el intraoperatorio se debe controlar cualquier condición que lleve a un aumento del estrés quirúrgico pues este a su vez genera hiperglucemia. Es fundamental el conocimiento de las insulinas, su farmacología y esquemas para mantener glucemias en el intraoperatorio en rangos normales. Se proponen diferentes algoritmos prácticos para el correcto y seguro manejo de la hiperglucemia en el perioperatorio. La atención debe continuarse en el posoperatorio definiendo continuidad de terapias insulínicas instauradas y el adecuado cuidado del paciente.

Palabras clave: Período perioperatorio, diabetes mellitus, hipoglucemiantes, insulina, hiperglucemia, Hipoglucemia. 


\section{Introducción}

a diabetes es definitivamente la endocrinopatía más común, datos indican que en el 2014 , el 8,6\% de la población padecía diabetes siendo responsable de al menos 3,7 millones de muertes por año. Se proyecta que para 2050 más del 30\% de la población tendrá diabetes, esto trae grandes implicaciones en términos de salud pública e impacto económico. Los datos y las perspectivas hacen que debamos conocer a profundidad el manejo y enfoque perioperatorio de esta población. La mitad de los diabéticos requerirá alguna cirugía a lo largo de su vida y uno de cada cuatro, cirugía cardíaca. Las estadísticas en cirugía cardiovascular describen que el 5,2\% son diabéticos no diagnosticados y esta cifra sube hasta $10 \%-28 \%$ en las cirugías no cardiacas. Lo anterior tiene un impacto importante dado que en cualquier tipo de procedimiento quirúrgico se muestran peores desenlaces en los diabéticos de novo comparados con los previamente diagnosticados, asimismo mayores tasas de reintubación, ventilación mecánica prolongada y aumento en mortalidad a un año[1],[2]. Nuestra intención con la presente revisión, es aportar al anestesiólogo una ayuda didáctica, práctica, clara y rápida, para la toma de decisiones frente al paciente con alteración del perfil glucémico en el perioperatorio.

\section{Hiperglucemia por estrés}

El estrés quirúrgico, el ayuno y la anestesia, alteran la regulación final del estado glucémico; se produce liberación de hormonas contrarreguladoras y de citoquinas proinflamatorias como IL-6 y el FNT (factor de necrosis tumoral). El cortisol aumenta la producción de glucosa hepática, estimula el catabolismo de las proteínas y promueve la gluconeogénesis. Las catecolaminas aumentan la secreción de glucagón e inhiben la liberación de insulina por las células Beta del páncreas[3]-[5].

Lo anterior en conjunto, aumenta la lipólisis, se produce una alta cantidad de ácidos grasos libres que inhiben la captación de glucosa estimulada por la insulina y limita la cascada de señalización intracelular en el músculo esquelético responsable de la actividad de transporte de glucosa. El FNT- $\alpha$ interfiere con la síntesis y translocación del transportador de glucosa 4, alterando el movimiento de glucosa en los tejidos periféricos. Se propicia entonces un estado alterado de la acción de la insulina, llevando a resistencia a la misma, siendo más pronunciado en el primer día posoperatorio, pudiendo persistir hasta por 21 días[3]-[5].

En la actualidad la carga preoperatoria de carbohidratos se está convirtiendo en una práctica frecuente, dado que puede contrarrestar el estado de resistencia a la insulina descrito anteriormente. Un ejemplo claro es el programa "Enhanced Recovery After Surgery" (ERAS) que promueve bebidas ricas en carbohidratos hasta 2 h antes de la cirugía. Esta práctica puede ayudar a evitar el estado catabólico asociado con la inanición, ha demostrado aumentar la sensibilidad a la insulina y a disminuir el riesgo de hiperglucemia posoperatoria, lo anterior especialmente en pacientes sometidos a cirugía abdominal mayor en quienes la carga de carbohidratos se ha asociado con una menor estancia hospitalaria[6].

\section{Relevancia del control glucémico}

Los resultados adversos son marcadamente elevados en aquellos pacientes con mal control glucémico incluyendo un aumento de más del $50 \%$ en la mortalidad, así mismo, incremento de infecciones respiratorias hasta 2,4 veces, infección sitio quirúrgico 2 veces, infección sitio urinario 3 veces, infarto agudo de miocardio 2 veces y lesión renal aguda 2 veces[7].

Per se, el solo hecho de presentar hiperglucemia intraoperatoria, con o sin antecedente o diagnóstico conocido de diabetes, ha demostrado aumentar la estancia hospitalaria, las infecciones del sitio quirúrgico, las infecciones urinarias y la mortalidad en cirugía no cardíaca. Adicionalmente, la cetoacidosis diabética o el estado hiperosmolar no cetónico se pueden presentar como complicaciones en estos pacientes. La hiperglucemia resultante es un factor de riesgo para sepsis posoperatoria, disfunción endotelial, isquemia cerebral y mala cicatrización[1]. O del modo contrario, la combinación de medicamentos hipoglucemiantes y el ayuno pueden generar una hipoglucemia severa, que podría pasar inadvertida durante la anestesia general o la sedación por un período de tiempo crítico, con desenlaces muy desfavorables para los pacientes[1]. La hipoglucemia no diagnosticada puede dar lugar a una serie de complicaciones neurológicas, entre ellas somnolencia, convulsiones, retraso en el despertar y según la duración, lesión neurológica irreversible, incremento de estancia en $\mathrm{UCl}$ e incluso la muerte[4],[8].

El uso de insulina incluido en el índice Lee (Score de Riesgo Cardiovascular para la valoración del riesgo cardiovascular en el paciente sometido a cirugía no cardíaca), ha sido implicada como factor de riesgo para muerte a 30 días y desenlaces cardiovasculares adversos siempre y cuando no se tenga control sobre la glucemia del paciente, ya que genera disfunción neurológica periférica por daño mitocondrial, producción de radicales libres y alteración de la función de las bombas sodio/ potasio ATPasa. Se calcula que aproximadamente, el $25 \%$ de los pacientes con diabetes tipo 1 y una tasa mayor de DM 2 (dependiendo del tiempo de evolución), pueden presentar cuadros de hiperglucemia perioperatorias. Esta situación predispone a mayor inestabilidad hemodinámica, retraso en el vaciamiento gástrico, retención o incontinencia urinaria, dismotilidad intestinal, pérdida de los reflejos vasomotores cutáneos, alteración de la perfusión cerebral, infartos cardíacos silentes, muerte súbita, entre otros[8]-[12].

La importancia del conocimiento de estas alteraciones, desenlaces y riesgos, está dado por la evidencia reciente, donde se sugiere que el manejo cuidadoso de los niveles de glucosa en pacientes sometidos a cirugías mayores, minimiza los efectos negativos y conlleva a mejores resultados metabólicos, infecciosos, neurológicos, cardiovasculares, renales, e incluso más recientemente descritos, efectos positivos sobre la memoria y la cognición a mediano y largo plazo. La tamización de pacientes hiperglucémicos basados en las características de riesgo y la identificación prequirúrgica puede modificar el enfoque, su manejo y así mismo los desenlaces[4],[9],[10],[13].

\section{Tamización}

La recomendación es realizar tamización con glucemia central o glucometrías durante el preoperatorio en todos los mayores de 45 años, aquellos entre 18 y 45 años deben ser tamizados si tienen alguno de los siguientes: familiar diabético de primer grado de consanguinidad, antecedentes de diabetes mellitus gestacional, antecedente de prediabetes, alguna condición que se asocia con resistencia a la insulina (consumo de 
esteroides, índice de masa corporal > 25, ovario poliquístico, inactividad física, hipertensión arterial, dislipidemia, acantosis nigricans, alteración endocrina, síndrome coronario agudo previo), y enfermedad cerebrovascular. $Y$ en aquellos menores de 18 años con IMC > p 85, más uno de los siguientes: familiar diabético de primer o segundo grado de consanguinidad, producto de embarazo con madre diabética gestacional, alteración que se relacione con resistencia a la insulina e inactividad física 14-16. Finalmente, en algunas revisiones incluyen como factor de riesgo las etnias de altas prevalencias como los hispánicos o asiáticos, situación que no aplica en nuestro medio[15],[16]. Por otro lado, la medición de los niveles $\mathrm{HbA} 1 \mathrm{c}$ los cuales reflejan el control glicémico durante los 8-12 semanas previas puede distinguir entre los pacientes con diabetes no diagnosticada de aquellos con hiperglucemia por estrés y sirve incluso como predictor postoperatorio[17],[18].

\section{Adecuado control glucémico preoperatorio}

Para los pacientes diabéticos la ADA (American Diabetes Association) recomienda una meta de $\mathrm{HbA} 1 \mathrm{c}$ menor de $7 \%$, sin embargo, se toleran cifras más laxas para ancianos con DM de larga data, porque no se ha visto que afecte en mayor medida los desenlaces microvasculares, por lo que en ellos una cifra entre 7 y $8 \%$ sería considerada en metas. El control glucémico intrahospitalario, al ser un estado más dinámico y puntual, se define como glucemias entre 140 y $180 \mathrm{mg} / \mathrm{dl}$; ya que, pese a las controversias, se conoce que tener metas más estrictas llevan a mayor riesgo de hipoglucemia con impacto negativo en la morbimortalidad[1],[19],[20]. Es prudente posponer el procedimiento electivo en caso de HbA1c muy elevada (> 9\%) o cifras de glucemia persistentemente por encima de 200 mg/dl, porque indican un pobre control glucémico y exponen al paciente a complicaciones metabólicas agudas en el perioperatorio. Cada $1 \%$ por encima de $9 \%$ aumenta el riesgo de desenlaces adversos en $40 \%$. Usando el mismo razonamiento, $\mathrm{HbA} 1 \mathrm{c}<5 \%$ indican probables episodios de hipoglucemia severa recurrente en un paciente tratado y también se recomienda posponer la cirugía electiva en este caso para optimizar el manejo[3],[20].

En el paciente quirúrgico en particular, es difícil saber el nivel óptimo de glucosa, es por esto que se han realizado grandes ensayos clínicos buscando la cifra ideal de glucemia, y al comparar pacientes con metas liberales (debajo de $180 \mathrm{mg} / \mathrm{dl}$ ), con grupos con metas estrictas (entre 80 y 100 mg/dl), no se encontró mejoría en los desenlaces posoperatorios, pero sí incrementó en el riesgo de complicaciones en el segundo grupo[21]-[22]. Por ello, se sustenta que el control intraoperatorio de estos pacientes debe estar basado en metas menores de $180 \mathrm{mg} / \mathrm{dl}$, ya sea con infusión de insulina o con esquemas de bolos subcutáneos y seguimiento estricto, idealmente cada institución de salud debe tener sus propios protocolos de manejo de la hiperglucemia perioperatoria[20]-[22]. Cualquier síntoma no explicado, independiente del nivel de glucemia, o una medición de la misma menor de $70 \mathrm{mg} / \mathrm{dl}$; es hipoglucemia en el paciente diabético y debe ser corregida de inmediato con $15 \mathrm{~g}$ de carbohidratos con control a los 15 minutos para verificar corrección. Aquellos pacientes con $\mathrm{HbA} 1 \mathrm{c}$ entre $6 \%-8 \%$ y con glicemia entre 100 $180 \mathrm{mg} / \mathrm{dl}$, pueden programarse para cirugía. Sin embargo, en los pacientes con $\mathrm{HbA} 1 \mathrm{c}<5 \% \mathrm{o}>9 \%$, glicemia $<60 \mathrm{mg} / \mathrm{dl}$ o > 300 mg/dl, o con coma hipoglucémico en el último mes o cetoacidosis, se debe posponer la cirugía. Por otro lado, pacientes con $\mathrm{HbA} 1 \mathrm{c}$ entre $5 \%-6 \%$, o entre $8 \%-9 \%$, o que presenten más de 2 episodios de hipoglicemia en la última semana o tengan riesgo de crisis hiperglicémicas, se debe considerar interconsulta rápida con médico tratante, e individualizar[23].

Durante la evaluación preoperatoria de pacientes con diabetes, es importante revisar el control glucémico y su tratamiento actual, además, de proporcionar al paciente instrucciones por escrito sobre cómo ajustar los medicamentos. En general, el día anterior a la cirugía los pacientes pueden continuar con todos los medicamentos[21], sin embargo, haremos una descripción de cada uno de ellos. La acidosis láctica inducida por metformina no es tan frecuente como se pensaba, pero son pacientes de riesgo para esta condición aquellos con infecciones severas, injuria renal aguda o los que requieran medios de contraste; y es en este grupo de pacientes en los cuales la metformina debe suspenderse[21]. La insulina basal requiere un ajuste de la dosis el día antes de la cirugía, su reducción puede ir entre el 50\% y el $80 \%$ según el estrés quirúrgico y el tipo de insulina. El manejo de los hipoglicemiantes orales en el preoperatorio de las cirugías electivas se debe realizar como se describe en la Tabla 1[24]-[27]. El uso de inhibidores DPP4 para el manejo de la población quirúrgica es seguro y deben ser continuados durante el perioperatorio[23].

En pacientes con diabetes tipo 1, es importante no interrumpir la administración de insulina basal para prevenir la aparición de cetoacidosis diabética[21]. El manejo de la insulinoterapia en el paciente diabético tipo 1 y 2 que será llevado a cirugía se presenta en la Figura 1. La dosis de insulina basal debe ser individualizada según el control glucémico del paciente, el tipo de cirugía y la duración de la misma, puesto que, en pacientes de alto riesgo de hipoglucemia, la dosis debe ser reducida hasta en $25 \%$ la noche previa a la cirugía. De manera similar, la dosis de insulina de acción intermedia debe reducirse a la mitad en todos los pacientes, ya que proporciona cobertura prandial para la comida del día, que probablemente se omitirá el día del procedimiento. Todas las dosis prandiales de acción corta se detienen hasta que el paciente reanude la ingesta alimentaria el día de la cirugía. El día anterior a la cirugía las insulinas de corta acción/preprandiales (aspart, lispo, glulisina e insulina regular) se deben continuar exactamente igual a como se están administrando. Para los pacientes tratados con preparaciones de insulina premezcladas que tienen niveles de glucosa en sangre en ayunas satisfactorios, la mitad de la dosis del componente basal se puede administrar como insulina de acción intermedia (NPH), sin embargo, si los pacientes tienen hiperglucemia en ayunas (> $200 \mathrm{mg} / \mathrm{dL}$ ), se puede administrar la mitad de la dosis de insulina premezclada antes de la cirugía[26]-[27]. En la Tabla 2 se describen los tipos de insulina, así como su farmacocinética.

Tal y como se mencionó al inicio de este artículo, la carga preoperatoria de carbohidratos se está convirtiendo en una práctica quirúrgica frecuente porque puede contrarrestar el estado de resistencia a la insulina y catabolismo que se producen debido al estrés y la inanición[15],[18],[27]. Entre algunos alimentos disponibles que podemos utilizar, que contienen aproximadamente $15 \mathrm{~g}$ de carbohidratos de acción rápida están: $118 \mathrm{ml}$ (media taza) de jugo de manzana, de naranja o leche descremada; una manzana o naranja pequeña o medio banano; 6 a 8 caramelos, una cucharada de miel mermelada o agua azucarada; 3 sobres de azúcar[28],[29]. 


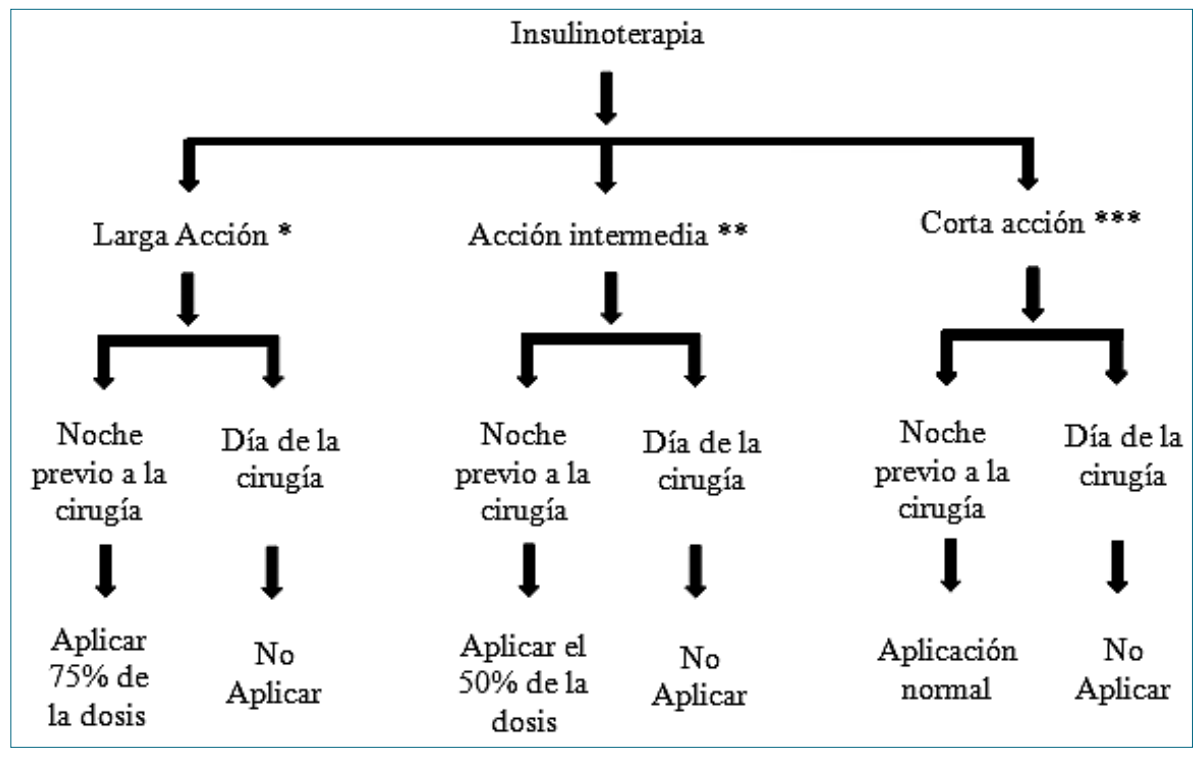

Figura 1. Ajuste de insulinoterapia en el periopertaorio. * Glargina, Detemir, Degludec / ** NPH / ** Regular, Aspart, Lispro, Glulisina. Fuente: Autores.

Entre 18 y 45 años, más uno de los siguientes

Menores de 18 años, con IMC > p 85, más uno de los siguientes

\section{Realizar a todos}

- Familiar diabético de primer grado de consanguinidad

- Antecedentes de diabetes mellitus gestacional

- Antecedente de prediabetes

- Condición que se asocia con resistencia a la insulina

- Consumo de esteroides

- Índice de masa corporal >25

- Ovario poliquístico

- Inactividad física

- Hipertensión arterial

- Dislipidemia

- Acantosis nigricans

- Alteración endocrina

- Síndrome coronario agudo previo

- Enfermedad cerebrovascular

- Familiar diabético de primer o segundo grado de consanguinidad

- Producto de embarazo con madre diabética gestacional

- Alteración que se relacione con resistencia a la insulina

- Inactividad física

- Acantosis nigricans

- Dislipidemia

- Hipertensión arterial

- Alteraciones endocrinas

Fuente: Autores. 


\begin{tabular}{|c|c|c|c|}
\hline Tipo & Prototipo & Día previo a cirugía & Día de la cirugía \\
\hline \multirow[t]{3}{*}{ Sulfonilureas } & Gliburida & No administrar & No administra \\
\hline & Glipizida & & \\
\hline & Glimepirida & & \\
\hline Biguanidas & Metformina & Administrar & Individualizar* \\
\hline \multirow[t]{2}{*}{ Tiazolidinedionas } & Rosiglitazona & Administrar & No administrar \\
\hline & Pioglitazona & & \\
\hline \multirow[t]{2}{*}{ Meglitinidas } & Repaglinida & No administrar & No administrar \\
\hline & Nateglinida & & \\
\hline \multirow[t]{2}{*}{ Inhibidores de la alfa glucosidasa } & Acarbosa & Administrar & No administrar \\
\hline & Miglitol & & \\
\hline \multirow[t]{3}{*}{ Inhibidores SGLT2 } & Canaglifozina & No administrar & No administrar \\
\hline & Dapaglifozina & & \\
\hline & Empaglifozina & & \\
\hline \multicolumn{4}{|c|}{ Miméticos de incretina** } \\
\hline \multirow[t]{2}{*}{ Agonistas GLP-1 } & Exenatide & Administrar & No administrar \\
\hline & Liraglutide & & \\
\hline \multirow[t]{4}{*}{ Inhibidores DPP4 } & Saxagliptina & Administrar & Administrar \\
\hline & Sitagliptina & & \\
\hline & Vildagliptina & & \\
\hline & Linagliptina & & \\
\hline
\end{tabular}

** GLP-1 = glucagón like peptide; DPP4 = dipeptidyl peptidasa 4; SGLT2 = sodio-glucosa cotransportador 2.

*Nota: si la cirugía es mínimamente invasiva, con bajo riesgo de sangrado, y no se va a recibir medio de contraste o hay bajo riesgo injuria renal o se puede retornar la vía oral rápidamente, podría administrarse la metformina previo a la cirugía.

Fuente: Autores. Adaptada de; Joshi GP, Chung F, Vann MA, et al. Society for Ambulatory Anesthesia consensus statement on perioperative blood glucose management in diabetic patients undergoing ambulatory surgery. Anesth Analg. 2010;111(6):1378-1387.

\section{Intraoperatorio}

Durante el intraoperatorio se debe realizar glucometría previa a la inducción anestésica a quienes tengan factores de riesgo y no hayan sido tamizados, además de monitoreo con glucemia periódica (al menos cada 1 a 2 h) en pacientes con alteraciones en la tamización o diabéticos. Es además fundamental optimizar volumen intravascular, mantener equilibrio electrolítico durante todo el perioperatorio como parte del manejo integral del paciente y en cirugías prolongadas monitoria del potasio cada 4 h[26],[30]. Cualquier condición que lleve a un aumento no controlado de estrés quirúrgico puede aumentar la glucemia, y es ahí donde radica la importancia de controlar factores como la temperatura, el dolor, la náusea, el vómito, entre otros. La hiperglucemia requiere corrección con esquema de insulina según la situación y asimismo se necesita proveer glucosa como sustrato para prevenir proteólisis, lipolisis y cetogénesis. Es así como la idea anterior nos plantea que los pacientes con hiperglucemia requieren controlar la misma con insulina, pero al mismo tiempo aporte de glucosa con dextrosa. La meta es mantener un rango de glucemia entre 140-180 mg/dl, lo cual cumple con el objetivo de disminuir morbimortalidad sin incremento de episodios de hipoglucemia[19],[26]. Es importante aclarar que, las complicaciones agudas de la diabetes deberán siempre ser descartadas en el contexto de hiperglucemia en el perioperatorio, considerando que hasta el $10 \%$ de las cetoacidosis diabéticas no cursan con glucemias mayores a $250 \mathrm{mg} / \mathrm{dl}$ (euglucémicas)[31].

Si durante el perioperatorio se supera el nivel de glucemia recomendado en dos mediciones separadas de 30 a 60 minutos o el paciente presenta cifras ascendentes, se debe considerar suministrar dosis de corrección con insulina cristalina o análogo de acción rápida. Es importante aclarar que la insulina cristalina tiene un inicio de acción a entre 15-30 minutos, con un pico a las 3 h y duración de acción de 6 h si se administra en bolo vía subcutánea, esto implica necesidad de monitoria estricta por riesgo de hipoglucemia. Los análogos de acción rápida tienen inicio de acción aproximadamente en 5 minutos, su pico de acción es a la hora y la duración media de 2 h[26].

Una primera clasificación que debe hacerse en el intraoperatorio de los pacientes diabéticos o hiperglucémicos es el requerimiento de infusión intravenosa o corrección subcutánea. Se propone que pacientes que vayan a ser sometidos a procedimientos de más de 4 h, con cambios hemodinámicos o uso inotrópicos, altas pérdidas sanguíneas o recambio alto de líquidos, cambios de temperatura importantes, o críticamente enfermos con previo manejo IV de insulina hospitalario, sean manejados con aplicación intravenosa, por la farmacocinética impredecible y variable de la vía subcutánea, así como la posibilidad de anticipar y titular cambios importantes de glucemia.

Insulina IV: en esta población, el seguimiento con gluco- 
Tabla 3. Tipos de insulina y farmacocinética

\begin{tabular}{|c|c|c|c|}
\hline Tipo de insulina y nombre genérico & Inicio de acción & Pico de acción & Duración de acción \\
\hline \multicolumn{4}{|l|}{ Rápida acción } \\
\hline - Aspart & $10-20 \mathrm{~min}$ & $40-50 \min$ & $3-5 h$ \\
\hline - Lispro & $15-30 \mathrm{~min}$ & $30-90 \min$ & $3-5 h$ \\
\hline - Glulisina & $20-30 \mathrm{~min}$ & $30-90 \min$ & $1-2,5 h$ \\
\hline \multicolumn{4}{|l|}{ Corta acción } \\
\hline - Regular & $0,5-1 \mathrm{~h}$ & $2-5 h$ & $5-8 h$ \\
\hline \multicolumn{4}{|l|}{ Intermedia } \\
\hline$-\mathrm{NPH}$ & $1-2 h$ & $4-12 h$ & $18-24 h$ \\
\hline \multicolumn{4}{|l|}{ Larga acción } \\
\hline - Glargina & $1-1,5 h$ & NA & $20-24 h$ \\
\hline - Detemir & $1-2 h$ & $6-8 h$ & $>24 \mathrm{~h}$ \\
\hline \multicolumn{4}{|l|}{ Muy larga acción } \\
\hline - Degludec & $30-90$ mins & NA & $42 \mathrm{~h}$ \\
\hline
\end{tabular}

* $\mathrm{R}=$ regular; $\mathrm{N}=$ neutra; $\mathrm{NPH}=$ neutral protamine hagedorn.

Fuente: Autores. Adaptada de; Joshi GP, Chung F, Vann MA, et al. Society for Ambulatory Anesthesia consensus statement on perioperative blood glucose management in diabetic patients undergoing ambulatory surgery. Anesth Analg. 2010;111(6):1378-1387.

metrías intraoperatorias e incluso en la Unidad de Cuidados Posanestésicos debe ser más riguroso y no sobrepasar nunca la hora de diferencia[3],[26],[32],[33]. La manera más sencilla y segura es la que mezcla $100 \mathrm{UI}$ de insulina hasta $100 \mathrm{ml}$ de SSN, de manera que se obtenga $1 \mathrm{UI} / \mathrm{ml}$. Se requiere monitorizar electrolitos principalmente potasio sérico y bicarbonato cada 4 h (mantener niveles de potasio entre 4-4,5). Simultáneamente, iniciar dextrosa para mantener un flujo metabólico basal de 4 $\mathrm{mg} / \mathrm{kg} / \mathrm{min}$, en promedio esto significa utilizar dextrosa al $10 \%$ a un promedio de 30-50 ml/h[18]. La tasa de infusión de la insulina dependerá de la glucemia obtenida; existe aún controversia respecto a dar o no un bolo inicial y luego continuar con una infusión por hora. Una forma práctica para empezar la infusión, es dividir la glucemia obtenida entre 100, y esa será la dosis en unidades a administrar por hora para el inicio[3],[18],[33], la propuesta de abordaje/titulación de la insulina y la dextrosa se presenta en la Tabla 3.

Insulina SC: contrario al grupo anterior, pacientes sin consumo previo de insulina, sin ayunos prolongados, que van a cirugías menores e incluso ambulatorias, no siempre se benefician de dicho esquema y su vía de elección puede ser la subcutánea. Además, el seguimiento intra y posoperatorio podría hacerse cada 2 h según los valores de glucometría[3],[32]. En este grupo de pacientes, cuando hay hiperglucemia, resulta útil hacer una clasificación rápida en dos sub-grupos: resistentes a la insulina: IMC mayor de 35; consumo de insulina previo mayor de 80 U/día; consumo crónico de esteroides. Sensibles a la insulina: mayores de 70 años; tasas de filtración glomerular menores de 35-45 ml/ min; IMC menor de 18[33]. Con base en la anterior clasificación y el nivel de glucemia se determinará la dosis a utilizar de insulina. Es así como el grupo con factores de riesgo para resistencia se le sumará 1-2 Ul adicional y al de riesgo de sensibilidad se le restará 1-2 UI. A continuación, se describe el esquema de corrección según el nivel de glucemia 3,33: entre 180 y 220 mg\%: 2-3 UI SC; 220 a 260 mg\%: 4 UI SC; 260 a 300 mg\%: 6 UI SC; 300 a 350 mg\%: 8 UI SC; 350 a 400 mg\%: 10 UI SC; más de 400 mg\%: 12 UI SC, considerar infusión.

Bombas de insulina: actualmente, reportes en Estados Unidos hablan de más de 400.000 pacientes que reciben infusión subcutánea continua de insulina (CSII). A pesar de que las bombas de insulina se usan predominantemente en el contexto ambulatorio, pacientes con este tipo de dispositivos cada vez más frecuentemente son llevados a procedimiento quirúrgi$\operatorname{cos[34]-[36].~En~la~actualidad,~no~hay~guías~específicas~periope-~}$ ratorias del abordaje de pacientes con bombas de insulina, esto crea limitantes importantes que pueden incluso poner en riesgo la vida de los pacientes[37]. La escasa cantidad de literatura al respecto hace que la comunicación entre endocrinología, anestesia, educador en diabetes, cirujano, paciente y sus familiares sean la clave para un manejo exitoso. Idealmente, se debe tener contacto directo con el médico endocrinólogo en el caso de cirugía electiva e incluso de cirugía emergente. Es importante hacer un paréntesis y mencionar que los CSII (por su sigla en inglés) son algunas veces manejado en asa cerrada, esto quiere decir que por medio de retroalimentación con mediciones continuas de glucemia subcutánea la bomba ajusta de manera automática la infusión de insulina según se requiera. Esto nunca debe limitar el uso de glucometría seriada para el monitoreo perioperatorio de los pacientes. Idealmente se debería crear una lista de chequeo que incluya tipo de bomba (CSII), tasa basal insulina, sitio implantación del cuerpo y estado de la bomba[37]. Una medida básica e importante en el caso de que no se logre comunicación con el médico endocrinólogo para modificar los parámetros de la bomba es decirle al paciente o a su familiar que apague la bomba y se realiza manejo como se especificó en la Tabla 3, con controles seriados de glucometría. Cabe anotar que la bomba debe ser protegida de golpes o líquidos.

Hipoglucemia: definida como glucemia menor de $70 \mathrm{mg} /$ $\mathrm{dl}$, se considera grave siempre que se encuentre por debajo de $40 \mathrm{mg} / \mathrm{dl}$ o que incluya compromiso marcado del paciente y su 


\begin{tabular}{|c|c|c|c|c|}
\hline \multirow[t]{2}{*}{ Glicemia } & \multirow[t]{2}{*}{ Inicio Infusión insulina } & \multicolumn{2}{|l|}{ Infusión de insulina } & \multirow[t]{2}{*}{$\begin{array}{l}\text { Infusión de DAD } 4 \mathrm{mg} / \\
\mathrm{kg} / \mathrm{min}\end{array}$} \\
\hline & & $\begin{array}{l}\text { Disminución } \\
<30 \mathrm{mg} / \mathrm{dL}\end{array}$ & $\begin{array}{l}\text { Disminución } \\
>30 \mathrm{mg} / \mathrm{dL}\end{array}$ & \\
\hline$>240$ & $0,1 \mathrm{U} / \mathrm{kg} / \mathrm{h}$ * & Aumentar $0,12 \mathrm{U} / \mathrm{kg} / \mathrm{h}$ & No cambio & No cambio \\
\hline 211 a 240 & $0,08 \mathrm{U} / \mathrm{Kg} / \mathrm{h}$ * & Aumentar $0,1 \cup / \mathrm{kg} / \mathrm{h}$ & No cambio & No cambio \\
\hline 181 a 210 & $0,05 \mathrm{U} / \mathrm{kg} / \mathrm{h}$ * & Aumentar $0,08 \mathrm{U} / \mathrm{kg} / \mathrm{h}$ & No cambio & No cambio \\
\hline 141 a 180 & No cambio* & No cambio & No cambio & No cambio \\
\hline 110 a 140 & No cambio* & Disminuir entre 0,5 y $1 \mathrm{U}$ por $\mathrm{h}$ & Suspender insulina & No cambio \\
\hline 91 a 109 & No iniciar/suspender* & $\begin{array}{l}\text { Reiniciar a la mitad de la tasa previa si } \\
\text { vuelve a ser mayor de } 180 \mathrm{mg} / \mathrm{dl}\end{array}$ & & No cambio \\
\hline 90 a 60 & No iniciar/suspender** & $\begin{array}{l}\text { Reiniciar a la mitad de la tasa previa si } \\
\text { vuelve a ser mayor de } 180 \mathrm{mg} / \mathrm{dl}\end{array}$ & & $\begin{array}{l}\text { Aumentar } \\
8 \mathrm{~g}(80 \mathrm{cc} \text { DAD 10\%) }\end{array}$ \\
\hline 59 a 40 & No iniciar/suspender $* * *$ & $\begin{array}{l}\text { Reiniciar a la mitad de la tasa previa si } \\
\text { vuelve a ser mayor de } 180 \mathrm{mg} / \mathrm{dl}\end{array}$ & $\begin{array}{l}\text { Aumentar } \\
15-30 \mathrm{~g}(150-300 \text { cc DAD } \\
10 \%)\end{array}$ & \\
\hline
\end{tabular}

*Medir glicemia en 1 hora.

** Medir glicemia en 30 minutos.

*** Medir glicemia en 15 minutos.

Fuente: Autores.

estado neurológico. El tratamiento de la hipoglucemia se realiza con glucagón del cual no siempre está disponible; se aplica 1 ampolla de 1 mg vía subcutánea o intramuscular. Si no se cuenta con glucagón se debe aplicar 15 a $30 \mathrm{~g}$ de glucosa lo que para efectos prácticos corresponde a 150 a $300 \mathrm{ml}$ de dextrosa al 10\%[3],[29],[33].

\section{Posoperatorio}

Es de gran importancia garantizar el adecuado control del dolor en el paciente con alteración glucémica en todo el perioperatorio, así como prevenir y tratar hábilmente las náuseas y vómito (PONV, por sus siglas en inglés), para facilitar el retorno de la función gastrointestinal temprana y la alimentación, de manera que se impacte sobre el estrés metabólico de la cirugía[38]. Con respecto a ese punto, vale la pena aclarar que uno de los pilares del manejo analgésico multimodal y simultáneamente de PONV es la dexametasona, que ha sido utilizado con temor en pacientes diabéticos por su efecto hiperglucemiante. Un metaanálisis reciente encontró que no había impacto negativo en la curación de las heridas quirúrgicas y que la elevación de la glucemia era de aproximadamente $14 \mathrm{mg} / \mathrm{dL}$ en pacientes no diabéticos y alrededor de $30 \mathrm{mg} / \mathrm{dL}$ en el diabético[39]. Las indicaciones de manejo glucémico deben continuar en la Unidad de Cuidados posanestésicos si el paciente va a permanecer allí. Aquellos que recibieron infusión de insulina y requieren continuarla en el POP deben ser trasladados a una unidad de alta dependencia donde se definirá el momento preciso para el cambio de esquema a subcutáneo, y se realizará una valoración por otras especialistas pertinentes. Es importante aclarar que, al cambiar la vía de administración a subcutánea, se debe iniciar 3 h antes de la suspensión de la infusión intravenosa, esto explicado por la farmacocinética de las insulinas[40].

\section{Conclusiones}

La DM es la endocrinopatía más frecuente, ampliamente distribuida en la población y con alto impacto en la morbimortalidad de los pacientes durante el perioperatorio. La adecuada tamización permite hacer diagnóstico en pacientes con DM de novo, optimizar el manejo previo a cirugía y llevar un control periódico durante el intraoperatorio para así mejorar los desenlaces en este grupo poblacional. El mal control glucémico se convierte en un predictor de malos desenlaces con riesgo tanto de hiper como de hipoglucemias inadvertidas durante la anestesia favoreciendo alteraciones neurológicas, procesos infecciosos, inestabilidad hemodinámica, eventos isquémicos, estancia en UCI e incluso la muerte. Las metas de control glucémico durante el perioperatorio más adecuadas corresponden a valores entre 140 - 180 mg/dl, el control periódico y el manejo con insulinas en bolos o en infusión asociada a DAD según esquemas institucionales, permite mantener estos valores y disminuir la morbimortalidad asociada. El correcto manejo, individualizando cada caso y definiendo el tratamiento con terapia insulínica intravenosa (infusión) o subcutánea es esencial, con una monitoria estricta y seguimiento adecuado.

Reconocimientos: Todos los autores: concepción del proyecto original, planificación del estudio, obtención de datos, interpretación de los resultados y redacción del manuscrito.

Asistencia para el estudio: ninguna.

Apoyo financiero y patrocinio: ninguno.

Conflictos de interés: ninguno.

Presentaciones: ninguna.

Agradecimientos: ninguno. 


\section{Referencias}

1. Sreedharan R, Abdelmalak B. Diabetes Mellitus: Preoperative Concerns and Evaluation. Anesthesiol Clin. 2018 Dec;36(4):58197. https://doi.org/10.1016/j.anclin.2018.07.007 PMID:30390780

2. Sosa García JO, García García AE, Soto Delgado KB, Romero González JP, Diaz Franco SD. Importancia del control glucémico durante el perioperatorio en pacientes con diabetes mellitus. Revista Mexicana de Anestesiología. 2020;43(1):48-52. https:// doi.org/10.35366/CMA201H.

3. Duggan EW, Carlson K, Umpierrez GE. Perioperative Hyperglycemia Management: an Update. Anesthesiology. 2017 Mar;126(3):547-60. https://doi.org/10.1097/ ALN.0000000000001515 PMID:28121636

4. Sudhakaran S, Surani SR. Guidelines for Perioperative Management of the Diabetic Patient. Surg Res Pract. 2015;2015:284063. https://doi.org/10.1155/2015/284063 PMID:26078998

5. Pérez de la Cruz A, Pérez Villares JM, Manzano Manzano F. Control de la hiperglucemia de estrés. Estado actual. Nutr Clin Med. 2019;13(1):1-18. https://doi.org/10.7400/NCM.2019.13.1.5070.

6. Ramírez-Cáceres C, Uzcátegui-Paz E, Lozano-Hernández R. Protocolo de rápida mejoría posoperatoria (RAMPO) en cirugía gastrointestinal. Cir Cir. 2019;87(2):151-7. https://doi.org/10.24875/ CIRU.18000470 PMID:30768060

7. Barker P, Creasey PE, Dhatariya K, Levy N, Lipp A, Nathanson $\mathrm{MH}$, et al.; Membership of the Working Party. Peri-operative management of the surgical patient with diabetes 2015: association of Anaesthetists of Great Britain and Ireland. Anaesthesia. 2015 Dec;70(12):1427-40. https://doi.org/10.1111/anae.13233 PMID:26417892

8. Hermanides J, Plummer MP, Finnis M, Deane AM, Coles JP, Menon DK. Glycaemic control targets after traumatic brain injury: a systematic review and meta-analysis. Crit Care. 2018 Jan;22(1):11. https://doi.org/10.1186/s13054-017-1883-y PMID:29351760

9. Miller JD, Richman DC. Preoperative Evaluation of Patients with Diabetes Mellitus. Anesthesiol Clin. 2016 Mar;34(1):155-69. https://doi.org/10.1016/j.anclin.2015.10.008 PMID:26927745

10. Gandhi H, Sarvaia A, Malhotra A, Acharya H, Shah K, Rajavat J. Effects of glargine insulin on glycemic control in patients with diabetes mellitus type II undergoing off-pump coronary artery bypass graft. Ann Card Anaesth. 2018 Apr-Jun;21(2):167-72. https://doi.org/10.4103/aca.ACA_128_17 PMID:29652278

11. Serhiyenko VA, Serhiyenko AA. Cardiac autonomic neuropathy: risk factors, diagnosis and treatment. World J Diabetes. 2018 Jan;9(1):1-24. https://doi.org/10.4239/wjd.v9.i1.1 PMID:29359025

12. Lee TH, Marcantonio ER, Mangione CM, Thomas EJ, Polanczyk CA, Cook EF, et al. Derivation and prospective validation of a simple index for prediction of cardiac risk of major noncardiac surgery. Circulation. 1999 Sep;100(10):1043-9. https://doi. org/10.1161/01.CIR.100.10.1043 PMID:10477528

13. Schricker $T$, Sato $H$, Beaudry $T$, Codere $T$, Hatzakorzian R, Pruessner JC. Intraoperative maintenance of normoglycemia with insulin and glucose preserves verbal learning after cardiac surgery. PLoS One. 2014 Jun;9(6):e99661. https://doi.org/10.1371/ journal.pone.0099661 PMID:24941010

14. Paulweber B, Valensi P, Lindström J, Lalic NM, Greaves CJ, McKee $M$, et al. A European evidence-based guideline for the prevention of type 2 diabetes. Horm Metab Res. 2010 Apr;42(S
01 Suppl 1):S3-36. https://doi.org/10.1055/s-0029-1240928 PMID:20391306

15. Levy N, Dhatariya K. Pre-operative optimisation of the surgical patient with diagnosed and undiagnosed diabetes: a practical review. Anaesthesia. 2019 Jan;74 Suppl 1:58-66. https://doi. org/10.1111/anae.14510 PMID:30604420

16. American Diabetes Association. 2. Classification and Diagnosis of Diabetes: Standards of Medical Care in Diabetes-2018. Diabetes Care. 2018 Jan;41 Suppl 1:S13-27. https://doi.org/10.2337/ dc18-S002 PMID:29222373

17. Mazurek JA, Hailpern SM, Goring T, Nordin C. Prevalence of hemoglobin A1c greater than $6.5 \%$ and $7.0 \%$ among hospitalized patients without known diagnosis of diabetes at an urban inner city hospital. J Clin Endocrinol Metab. 2010 Mar;95(3):1344-8. https://doi.org/10.1210/jc.2009-1151 PMID:20080838

18. Cheisson $G$, Jacqueminet $S$, Cosson $E$, Ichai $C$, Leguerrier $A M$, Nicolescu-Catargi B, et al.; working party approved by the French Society of Anaesthesia and Intensive Care Medicine (SFAR), the French Society for the study of Diabetes (SFD). Perioperative management of adult diabetic patients. Intraoperative period. Anaesth Crit Care Pain Med. 2018 Jun;37 Suppl 1:S21-5. https://doi.org/10.1016/j.accpm.2018.02.018 PMID:29555547

19. Finfer $S, C$ hittock $D R$, Su SY, Blair D, Foster D, Dhingra $V$, et al.; NICE-SUGAR Study Investigators. Intensive versus conventional glucose control in critically ill patients. N Engl J Med. 2009 Mar;360(13):1283-97. https://doi.org/10.1056/NEJMoa0810625 PMID:19318384

20. Kuzulugil D, Papeix G, Luu J, Kerridge RK. Recent advances in diabetes treatments and their perioperative implications. Curr Opin Anaesthesiol. 2019 Jun;32(3):398-404. https://doi. org/10.1097/ACO.0000000000000735 PMID:30958402

21. Simha V, Shah P. Perioperative Glucose Control in Patients With Diabetes Undergoing Elective Surgery. JAMA. 2019 Jan;321(4):399-400. https://doi.org/10.1001/jama.2018.20922 PMID:30615031

22. Siddiqui KM, Asghar MA, Khan MF, Khan FH. Perioperative glycemic control and its outcome in patients following open heart surgery. Ann Card Anaesth. 2019 Jul-Sep;22(3):260-4. https:// doi.org/10.4103/aca.ACA_82_18 PMID:31274486

23. Cheisson $G$, Jacqueminet $S$, Cosson $E$, Ichai $C$, Leguerrier $A M$, Nicolescu-Catargi B, et al.; working party approved by the French Society of Anaesthesia and Intensive Care Medicine (SFAR), the French Society for the study of Diabetes (SFD). Perioperative management of adult diabetic patients. Preoperative period. Anaesth Crit Care Pain Med. 2018 Jun;37 Suppl 1:S9-19. https:// doi.org/10.1016/j.accpm.2018.02.020 PMID:29559406

24. Milder DA, Milder TY, Kam PC. Sodium-glucose co-transporter type-2 inhibitors: pharmacology and peri-operative considerations. Anaesthesia. 2018 Aug;73(8):1008-18. https://doi. org/10.1111/anae.14251 PMID:29529345

25. Lorenzo-González C, Atienza-Sánchez E, Reyes-Umpierrez D, Vellanki P, Davis GM, Pasquel FJ, et al. Safety and efficacy of ddp4-inhibitors for management of hospitalized general medicine and surgery patients with type 2 diabetes. Endocr Pract. 2020 Apr;26(7):722-8. https://doi.org/10.4158/EP-2019-0481 PMID:32339032

26. Joshi GP, Chung F, Vann MA, Ahmad S, Gan TJ, Goulson DT, et al.; Society for Ambulatory Anesthesia. Society for Ambulatory Anesthesia consensus statement on perioperative blood glu- 
cose management in diabetic patients undergoing ambulatory surgery. Anesth Analg. 2010 Dec;111(6):1378-87. https://doi. org/10.1213/ANE.0b013e3181f9c288 PMID:20889933

27. Morimoto $Y$, Kinugawa T, Hayashi M, lida T, Yamamoto T. Effects of preoperative oral carbohydrate intake on catabolism, nutrition and adipocytokines during minor surgery: A randomized, prospective, controlled clinical phase II trial. PLoS One. 2019 May;14(5):e0216525. https://doi.org/10.1371/journal. pone.0216525 PMID:31083704

28. Cornelius BW. Patients With Type 2 Diabetes: Anesthetic Management in the Ambulatory Setting: Part 2: Pharmacology and Guidelines for Perioperative Management. Anesth Prog. 2017;64(1):39-44. https://doi.org/10.2344/anpr-64-01-02 PMID:28128657

29. Thompson ME, Noel MB. Issues in Nutrition: carbohydrates. FP Essent. 2017 Jan;452:26-30. https://doi.org/10.1515/rjdnmd-2017-0021 PMID:28092151

30. Dogra P, Jialal I. Diabetic Perioperative Management[Updated 2020 Jan 22] StatPearls [Internet]Treasure Island (FL): StatPearls Publishing; 2020 Jan., Available from https://www.ncbi.nlm.nih. gov/books/NBK540965/

31. Modi A, Agrawal A, Morgan F. Euglycemic Diabetic Ketoacidosis: A Review. Curr Diabetes Rev. 2017;13(3):315-21. https://doi.org/ 10.2174/1573399812666160421121307 PMID:27097605

32. Tankel J, Zimmerman F, De Jong A, Martin-Loeches I, Einav $S$. From the ICU to the operating room: how to manage the patient? Curr Opin Anaesthesiol. 2020 Apr;33(2):139-45. https:// doi.org/10.1097/ACO.0000000000000785 PMID:31503036

33. Duggan E, Chen $Y$. Glycemic Management in the Operating Room: Screening, Monitoring, Oral Hypoglycemics, and Insulin Therapy. Curr Diab Rep. 2019 Nov; 19(11):134. https://doi. org/10.1007/s11892-019-1277-4 PMID:31749027
34. Cook CB, Boyle ME, Cisar NS, Miller-Cage V, Bourgeois P, Roust $L R$, et al. Use of continuous subcutaneous insulin infusion (insulin pump) therapy in the hospital setting: proposed guidelines and outcome measures [Erratum in: Diabetes Educ. 2006;32:130]. Diabetes Educ. 2005 Nov-Dec;31(6):849-57. https://doi. org/10.1177/0145721705281563 PMID:16288092

35. Leonhardi BJ, Boyle ME, Beer KA, Seifert KM, Bailey M, Miller-Cage $V$, et al. Use of continuous subcutaneous insulin infusion (insulin pump) therapy in the hospital: a review of one institution's experience. J Diabetes Sci Technol. 2008 Nov;2(6):948-62. https://doi.org/10.1177/193229680800200605 PMID:19885284

36. Noschese ML, DiNardo MM, Donihi AC, Gibson JM, Koerbel $\mathrm{GL}$, Saul M, et al. Patient outcomes after implementation of a protocol for inpatient insulin pump therapy. Endocr Pract. 2009 Jul-Aug;15(5):415-24. https://doi.org/10.4158/EP09063.ORR PMID:19491071

37. Nassar AA, Boyle ME, Seifert KM, Beer KA, Apsey HA, Schlinkert RT, et al. Insulin pump therapy in patients with diabetes undergoing surgery. Endocr Pract. 2012 Jan-Feb;18(1):49-55. https:// doi.org/10.4158/EP11157.OR PMID:21803711

38. Lloyd $\mathrm{H}$. Perioperative care of the adult diabetic patient. J Perioper Pract. 2020 Dec;30(12):372-7. https://doi. org/10.1177/1750458920915660 PMID:32301384

39. Toner AJ, Ganeshanathan V, Chan MT, Ho KM, Corcoran TB. Safety of perioperative glucocorticoids in elective noncardiac surgery: a systematic review and meta-analysis. Anesthesiology. 2017 Feb;126(2):234-48. https://doi.org/10.1097/ ALN.0000000000001466 PMID:27922839

40. Tickoo M. The Long and Winding Road to Personalized Glycemic Control in the Intensive Care Unit. Semin Respir Crit Care Med. 2019 Oct;40(5):571-9. https://doi.org/10.1055/s-0039-1697603 PMID:31826258 